\title{
Biophysical Insights into Cystic Fibrosis Based on Electromagnetic Resonances in CFTR Proteins
}

\author{
Irena Cosic ${ }^{1,2}$, Vasilis Paspaliaris ${ }^{3}$, Drasko Cosic ${ }^{2}$
}

\author{
${ }^{1}$ RMIT University, Melbourne, Australia \\ ${ }^{2}$ AMALNA Consulting, Black Rock, Australia \\ ${ }^{3}$ Tithon Biotech Inc, San Diego, USA
}

\begin{abstract}
The cystic fibrosis is genetic disease characterised by build-up of thick mucus in the lungs, which causes difficulties in breathing. It is caused by mutations in CFTR protein. Here, we utilised the Resonant Recognition Model (RRM), which proposes that proteins specific activity is characterised by specific frequencies (wavelengths) of electromagnetic radiation. Using the RRM approach, we have identified the electromagnetic frequency (wavelength) characterising the healthy CFTR proteins, while the amplitude at this frequency is diminishing in mutated CFTR proteins. Thus, the identified characteristic frequency (wavelength) for heathy CFTR proteins could be proposed to be critical for healthy functioning of CFTR proteins and for differentiating between healthy and malfunctioning CFTR proteins related to cystic fibrosis. In addition, using the RRM approach we achieved the possible explanation on how specific temperature of $27-28^{\circ} \mathrm{C}$ can restore the healthy function in mutated CFTR proteins.
\end{abstract}

Keywords: Protein Electromagnetic Resonances, Cystic Fibrosis, CFTR Proteins, Resonant Recognition Model, Molecular Modelling

\begin{abstract}
Introduction
The cystic fibrosis is genetic disease characterised by build-up of thick mucus in the lungs, which causes difficulties in breathing, can cause progressive lung damage and premature mortality. Pulmonary flareups of this lung disease often require hospitalisation for intensive treatment. The standard treatment for cystic fibrosis is usage of antibiotics to reduce loss of lung function and thus improve survival [Schultz et al., 2019].
\end{abstract}

This disease is caused by mutations in CFTR protein, which is transmembrane conductance regulatory protein. An improved understanding of CFTR structure and consequences of CFTR gene mutations have allowed the development of novel therapies based on gene editing: "Some strategies are mutation specific and have already reached clinical development; some strategies include a read-through of the specific premature termination codons (readthrough therapies, nonsense mediated decay pathway inhibitors for Class I mutations); correction of CFTR folding and trafficking to the apical plasma membrane (correctors for Class II mutations); and an increase in the function of CFTR channel (potentiators therapy for Class III mutations and any mutant with a residual function located at the membrane). Other therapies that are in preclinical development are not mutation specific and include gene therapy to edit the genome and stem cell therapy to repair the airway tissue. These strategies that are directed at the basic $\mathrm{CF}$ defects are now revolutionizing the treatment for patients and should positively impact their survival rates" [Pranke et al., 2019].

While classical treatment with antibiotics is only helping with symptoms the novel approaches using personalised gene editing are much more promising. However, most of these novel approaches are still in clinical trial stages, complicated and very expensive. Thus, there is need for simplified and cheaper approach for understanding of cystic fibrosis and novel drug development. Here, we tried to understand cystic fibrosis from an innovative biophysical angle, particularly analysing biophysical basis of CFTR protein activity, whose malfunctioning is the cause of this disease.

The CFTR protein is cell membrane channel protein that indirectly controls the flow of $\mathrm{H}_{2} \mathrm{O}$ and $\mathrm{CI}^{-}$ions in and out of cells inside lungs through number of steps. When CFTR protein is malfunctioning, these ions cannot flow out of the cell due to blocked channel causing cystic fibrosis, characterised by the build-up of thick mucus in the lungs, which makes it difficult for person to breath [O'Sullivan and Freedman, 2009]. Malfunctioning of CFTR protein is caused by mutations in both copies of CFTR gene. The most common mutation that causes the cystic 
fibrosis is deletion of amino acid, phenylalanine $(\mathrm{F})$ at $508^{\text {th }}$ position of the CFTR protein. Several other mutations in the CFTR gene can occur, and different mutations cause different defects in the CFTR protein, sometimes causing a milder or more severe disease. The most common CFTR protein mutations that cause the cystic fibrosis are: $\Delta \mathrm{F} 508, \mathrm{G} 542 \mathrm{X}$, G551D, N1303K and W1282X [O'Sullivan and Freedman, 2009]. In addition, the number of experiments in cell cultures have shown that specific temperature between $27-28^{\circ} \mathrm{C}$ can restore effect of F508 deletion in CFTR proteins [Gomes-Alves et al., 2009; Rennolds et al., 2008; Wilke et al., 2012]. The fact that temperature can restore the function of mutated proteins gave us an idea that activity of CFTR proteins can be manipulated by energy levels in the environment, which might be done using electromagnetic radiation. To understand such process, we used the Resonant Recognition Model (RRM), which proposes that protein activity and selective interactions are based on electromagnetic resonances between interacting macromolecules [Cosic, 1994; Cosic, 1995; Cosic, 1997; Cosic et al., 2015; Cosic et al., 2016]. For that purpose, we analysed CFTR proteins and their mutants using the RRM approach.

\section{Methods and Materials \\ Resonant Recognition Model (RRM)}

The Resonant Recognition Model (RRM), which we applied here to CFTR proteins, is biophysical approach that can analyse protein biological functions and their selective interactions. The RRM model has been extensively explained within Appendix.

The selectivity and specificity of protein biological functions, as well as protein 3D structure are written within the protein sequence of amino acids. The RRM model proposes that protein functions and protein selective interactions are based on electromagnetic resonances between protein and its target. These resonances are within the range of far infra-red, infra-red, visible and ultra-violet light.

Here, we have applied the RRM to analyse protein sequences using the following steps:

- Amino acid sequence is converted into the sequence of numbers by assigning to each amino acid corresponding value of electron ion interaction potential (EIIP), which describes energies of free electrons in each amino acid residue and is related to ionisation potential [Pirogova and Cosic, 2002]. Such numerical sequence caries the same information as sequence of amino acids, but also represents distribution of free energies along the protein.

- This numerical sequence is then converted into numerical spectra using the Fast Fourier Transform (FFT). As the distance between amino acid residues in the protein backbone is $3.8 \AA$, it can be assumed that the points in the numerical sequence are equidistant and thus they are set at an arbitrary value of $\mathrm{d}=1$. Consequently, the maximum frequency in the spectrum is $\mathrm{F}=1 / 2 \mathrm{~d}=0.5$. The total number of points in the sequence influences the resolution of the spectrum, for $\mathrm{N}$-point sequence the resolution in the spectrum is equal to $1 / \mathrm{N}$. The $\mathrm{n}$-th point in the spectral function corresponds to the frequency $\mathrm{f}=\mathrm{n} / \mathrm{N}$.

- In order to extract common spectral characteristics of proteins having the same or similar biological function, the multiple crossspectral function is used. Peak frequencies in such multiple cross-spectral function presents common frequency components for all sequences analysed and through large number of already published examples, are found to characterise common biological function of analysed proteins [Cosic, 1994; Cosic, 1995; Cosic, 1997]. Signalto-noise ratio $(\mathrm{S} / \mathrm{N})$ for each peak is used as measure of similarity between analysed sequences and is calculated as the ratio between signal intensity at the specific frequency and the mean value over the whole spectrum.

- It has been shown that interacting proteins and their targets share the same characteristic frequency and thus, the RRM frequencies characterize protein function through resonant recognition and interaction with its target [Cosic, 1994; Cosic, 1997].

- The biophysical meaning of these characteristic RRM frequencies is based on the charge moving through the protein backbone, passing different energy stages, due to different amino acid side groups and producing enough conditions for specific electromagnetic radiation or absorption. The frequency of this radiation depends on the charge velocity, which is proposed by RRM to be at $7.87 \times 10^{5} \mathrm{~m} / \mathrm{s}$ [Cosic, 1994; Cosic, 1997]. For this velocity and with the distance between amino acids in protein backbone of $3.8 \AA$, the wavelength of electromagnetic radiation related to protein functions/interactions was estimated to be in the range between $400 \mathrm{~nm}$ and $40000 \mathrm{~nm}$, including far infra-red, infra-red, visible and ultra-violet light.

- We have used here the following formula, as proposed by RRM and explained within supplementary material section, to convert between numerical RRM frequencies and corresponding electromagnetic radiation: $\lambda=\mathrm{K} / \mathrm{f}_{\mathrm{rrm}}$

where $\lambda$ is the wavelength of light irradiation in $\mathrm{nm}$, which can influence specific process, $\mathrm{f}_{\mathrm{rrm}}$ is $\mathrm{RRM}$ numerical frequency and $\mathrm{K}$ is coefficient of this linear correlation. This formula has been supported by number of published experimental 
results including: laser light growth promotion of cells, chymotrypsin activation achieved by laser light radiation, activation of highly homologous plant photoreceptors which, although being very homologous, absorb different wavelengths of light and photo activated proteins, e.g. rhodopsin, flavodoxin, etc [Cosic, 1994; Cosic, 1997].

Using this formula has been also tested experimentally on L-Lactate Dehydrogenase activity [Vojisavljevic et al., 2007], on experimental measurements of photon emission from dying melanoma cells [Dotta et al., 2014], on photon emission from lethal and non-lethal Ebola strains [Murugan et al., 2014], as well as on classic signalling pathway, JAK-STAT composed of nine sequential protein interactions [Karbowski et al., 2015]. Even more, the RRM model, for the first time, explains how and why external blue light can be used in the treatment of Crigler-Najjar syndrome [Cosic and Cosic, 2016].

Here, we have applied the RRM approach for the first time to CFTR proteins with the aim to identify the characteristic numerical RRM frequency for healthy CFTR proteins and how mutations affect this frequency and consequently affect CFTR protein activity causing cystic fibrosis. We also have calculated electromagnetic wavelength for this numerical RRM frequency that is critical for healthy functioning of CFTR proteins. Based on possibility that specific wavelength of electromagnetic radiation is critical for healthy functioning of CFTR proteins and using the fact that specific temperature of 27$28^{\circ} \mathrm{C}$ recovers the functionality of mutated CFTR proteins, we have extended here the RRM approach to correlate RRM frequencies with temperature.

\section{Materials}

The following twelve CFTR proteins from UniProt database have been used here for RRM analysis as described above:

>sp|Q00552|CFTR_CAVPO Cystic fibrosis transmembrane conductance regulator OS=Cavia porcellus $\mathrm{OX}=10141 \mathrm{GN}=\mathrm{CFTR} \mathrm{PE}=2 \mathrm{SV}=2$

$>$ sp|Q2QLE5|CFTR_PANTR Cystic fibrosis transmembrane conductance regulator $\mathrm{OS}=\mathrm{Pan}$ troglodytes $\mathrm{OX}=9598 \mathrm{GN}=\mathrm{CFTR} \mathrm{PE}=3 \mathrm{SV}=1$

$>$ sp|Q5U820|CFTR_CANLF Cystic fibrosis transmembrane conductance regulator $\mathrm{OS}=\mathrm{Canis}$ lupus familiaris $\mathrm{OX}=9615 \mathrm{GN}=\mathrm{CFTR} \mathrm{PE}=2 \mathrm{SV}=2$
>sp|P26361|CFTR_MOUSE Cystic fibrosis transmembrane conductance regulator $\mathrm{OS}=$ Mus musculus $\mathrm{OX}=10090 \mathrm{GN}=\mathrm{Cftr} \mathrm{PE}=1 \mathrm{SV}=2$

$>$ sp|P13569|CFTR_HUMAN Cystic fibrosis transmembrane conductance regulator $\mathrm{OS}=\mathrm{Homo}$ sapiens $\mathrm{OX}=9606 \mathrm{GN}=\mathrm{CFTR} \mathrm{PE}=1 \mathrm{SV}=3$

>sp|Q6PQZ2|CFTR_PIG Cystic fibrosis transmembrane conductance regulator $\mathrm{OS}=$ Sus scrofa $\mathrm{OX}=9823 \mathrm{GN}=\mathrm{CFTR} \mathrm{PE}=2 \mathrm{SV}=1$

$>$ sp|Q00554|CFTR_RABIT Cystic fibrosis transmembrane conductance regulator OS=Oryctolagus cuniculus OX=9986 GN=CFTR $\mathrm{PE}=2 \mathrm{SV}=4$

>sp|Q2QLA3|CFTR_HORSE Cystic fibrosis transmembrane conductance regulator $\mathrm{OS}=$ Equus caballus $\mathrm{OX}=9796 \mathrm{GN}=\mathrm{CFTR} \mathrm{PE}=3 \mathrm{SV}=1$

$>$ sp|P35071|CFTR_BOVIN Cystic fibrosis transmembrane conductance regulator $\mathrm{OS}=\mathrm{Bos}$ taurus $\mathrm{OX}=9913 \mathrm{GN}=\mathrm{CFTR} \mathrm{PE}=2 \mathrm{SV}=2$

>sp|Q2IBF6|CFTR_GORGO Cystic fibrosis transmembrane conductance regulator $\mathrm{OS}=$ Gorilla $\mathrm{OX}=9595 \mathrm{GN}=\mathrm{CFTR} \mathrm{PE}=3 \mathrm{SV}=1$

$>$ sp|Q00555|CFTR_SHEEP Cystic fibrosis transmembrane conductance regulator $\mathrm{OS}=\mathrm{Ovis}$ aries $\mathrm{OX}=9940 \mathrm{GN}=\mathrm{CFTR}$ PE$=1 \mathrm{SV}=2$

$>$ sp|P34158|CFTR_RAT Cystic fibrosis transmembrane conductance regulator OS=Rattus norvegicus $\mathrm{OX}=10116 \mathrm{GN}=\mathrm{Cftr} \mathrm{PE}=1 \mathrm{SV}=3$.

\section{Results and Discussion}

Here, we have applied the RRM model, described above and within supplementary material section, to understand cystic fibrosis from biophysical angle, by analysing biophysical bases of CFTR protein activity and then understanding why some mutated CFTR proteins are causing cystic fibrosis. To achieve that, we have applied the RRM approach to CFTR proteins to find out the characteristic RRM frequency of healthy CFTR protein function and how this RRM frequency changes in malfunctioning mutated CFTR proteins. For this purpose, we analysed twelve healthy CFTR proteins from UniProt database, as listed above. We have found the most prominent common frequency at $\mathrm{f} 1=0.0444 \pm 0.0007$ with high signal-to-noise ratio of 863 , as presented in Figure 1. This result indicates that frequency $\mathrm{f} 1$ is characterising CFTR protein's healthy activity. According to conversion formula between numerical RRM frequency into the electromagnetic wavelength in nanometres $(\mathrm{nm})$ the frequency $\mathrm{f1}$ corresponds to electromagnetic radiation of $4527 \mathrm{~nm}$, which is within far infra-red spectrum. 


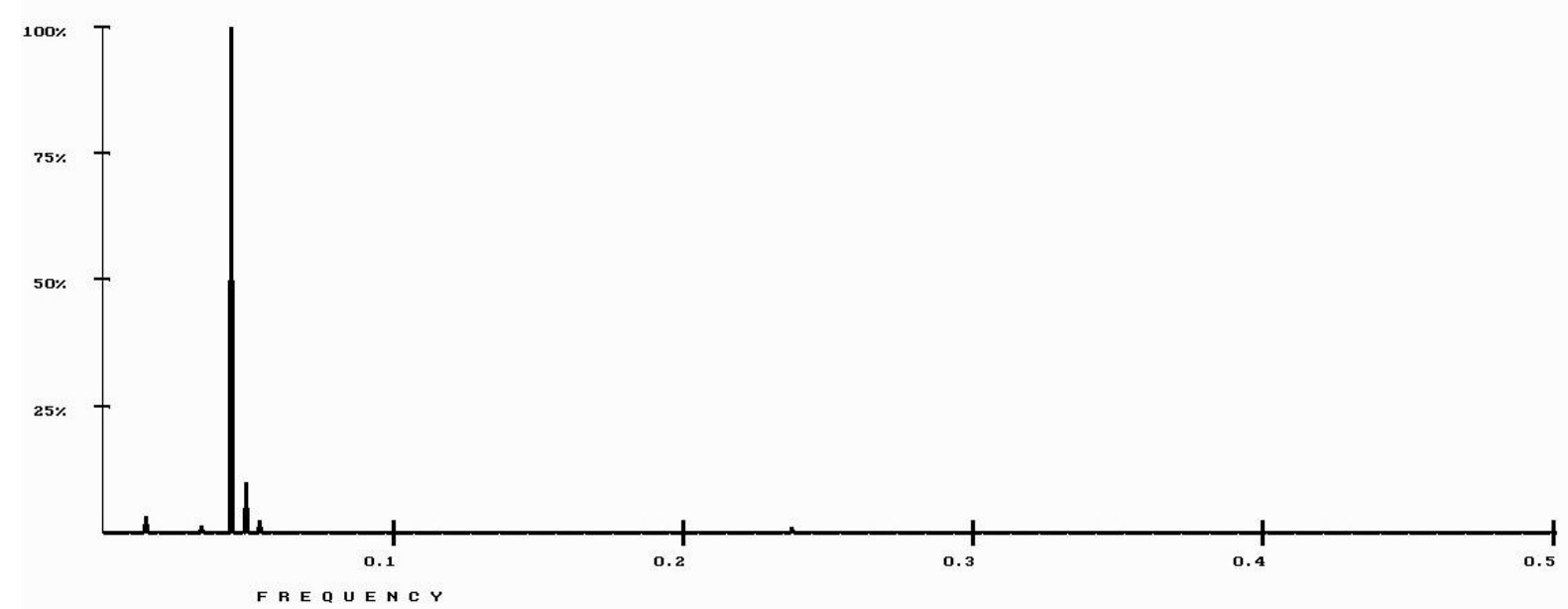

Figure 1. RRM cross-spectrum of twelve healthy CFTR proteins. The common RRM characteristic frequency is at $\mathrm{f} 1=0.0444 \pm 0.0007$, with signal-to-noise ratio of 863 . Numerical frequency f1 corresponds to electromagnetic radiation wavelength of $4527 \mathrm{~nm}$. X-axis represents numerical RRM frequency, which could reach maximum of 0.5 as explained within text. Y-axis represents percentages of maximum peak, which could reach maximum of $100 \%$. When we analysed same twelve CFTR proteins, but with deletion F508, using the RRM, the same most prominent common frequency has been found at $\mathrm{f} 1=0.0444 \pm 0.0007$, but with much smaller signal-to-noise ratio of 686 . This result points out that mutated proteins have much lower amplitude at RRM characteristic frequency f1, indicating lower activity in mutated proteins and thus could explain why CFTR protein with F508 deletion is malfunctioning. When we analysed same group of CFTR proteins, but with simultaneous deletion F508 and most common mutations: G542X, G551D, N1303K and W1282X, using the RRM, the same most prominent common frequency has been found at $\mathrm{f} 1=0.0444 \pm 0.0007$. The signal-to-noise ratio at the same frequency $\mathrm{f} 1 \mathrm{has}$ diminished even further to value of 534, indicating even further diminishing activity of such mutated CFTR protein. All these results are indicating that frequency $\mathrm{f} 1$ is critical for normal, healthy functioning of CFTR proteins.

It has been shown, that specific temperature between $27-28^{\circ} \mathrm{C}$ can restore the function of mutated CFTR protein with F508 deletion, by not correcting F508 deletion, but allowing CFTR to access the plasma membrane and thus allow the limited function to be performed. This fact gave us an idea that activity of the CFTR proteins can be manipulated by temperature, which could be related to energy levels of radiation possibly electromagnetic in nature. On the other hand, the RRM model proposes that specificity of protein activity is characterised by different wavelengths of electromagnetic radiation within the range of far infra-red, infra-red, visible and ultra-violet light. Thus, we propose here that temperature influence on activity of CFTR proteins and their mutants, possibly other proteins in general, can be related to electromagnetic radiation of wavelengths specific for protein function, as proposed by RRM.
To explain how this specific temperature can restore the function of F508 deletion in CFTR proteins, we introduced the approximate relationship between temperature and numerical RRM characteristic frequencies. If we consider that temperature of roughly $43-44^{\circ} \mathrm{C}$ is maximal temperature that most of the proteins can normally function, we assign maximum possible numerical RRM frequency of 0.5 to this temperature. On the other hand, the optimal temperature for cell growth is roughly between 36$37^{\circ} \mathrm{C}$ which we assigned to the main numerical RRM growth frequency, previously calculated at approximately 0.3 [Cosic, 1997]. We considered the general rule of physics that higher frequency corresponds to higher energy and consequently to higher temperature, as well as that relationship between energy and electromagnetic frequency is linear. Using this linearity, we can propose the following relationship between numerical RRM frequency and temperature, as presented in Table 1.

\begin{tabular}{|l|l|l|l|l|l|l|l|}
\hline RRM Frequency & 0.5 & 0.4 & 0.3 & 0.2 & 0.1 & 0.05 & 0.0444 \\
\hline Temperature $\left({ }^{\circ} \mathrm{C}\right)$ & $43-44$ & $39.5-40.5$ & $36-37$ & $32.5-33.5$ & $29-30$ & $27.25-28.25$ & $27-28$ \\
\hline
\end{tabular}

Table 1. Estimated relationship between RRM frequency and temperature

When relationship between numerical RRM frequency and temperature is introduced, as described above, the RRM characteristic frequency of $\mathrm{f} 1=0.0444 \pm 0.0007 \quad(4527 \mathrm{~nm}) \quad$ corresponds to temperature of $27-28^{\circ} \mathrm{C}$, as presented in Table 1 . This could mean that temperature of $27-28^{\circ} \mathrm{C}$ is related to far infra-red radiation of $4527 \mathrm{~nm}$, proposed by RRM to characterise normal functioning of CFTR proteins. This result can give an explanation on how specific temperature of $27-28^{\circ} \mathrm{C}$ can restore functioning of 
mutated CFTR proteins.

\section{Conclusion}

The cause of cystic fibrosis, which is genetic disease affecting lung function, are specific mutations within CFTR proteins. Here, we have applied the Resonant Recognition Model (RRM), which is based on electromagnetic resonant energy transfer between interacting proteins, to CFTR proteins. We have identified the characteristic of healthy CFTR proteins to be at numeric RRM frequency of $\mathrm{f} 1=0.0444 \pm 0.0007$, that is according to $\mathrm{RRM}$ principles corresponding to electromagnetic wavelength of $4527 \mathrm{~nm}$. We have found, using the cross spectra function, that the amplitude at this characteristic numerical frequency is diminishing in mutated CFTR proteins. This finding could explain how and why mutated CFTR proteins cannot achieve expected biological function and thus cause cystic fibrosis. In addition, using the RRM approach, we achieved possible explanation on how specific temperature of $27-28^{\circ} \mathrm{C}$ can restore effect of F508 deletion in CFTR proteins. Having these results in mind, some novel approaches in treating cystic fibrosis could be investigated: use of optical nanoparticles that can resonate with characteristic electromagnetic wavelength of $4527 \mathrm{~nm}$, photo therapy with far infra-red electromagnetic wavelength of $4527 \mathrm{~nm}$ or design of bioactive peptides based on characteristic numerical RRM frequency f1. All proposed therapies could be useful in restoring activity of mutated CFTR proteins in treatment of cystic fibrosis.

\section{References}

Cosic I. 1994. Macromolecular Bioactivity: Is it Resonant Interaction between Macromolecules? Theory and Applications. IEEE Trans on Biomedical Engineering 41:1101-1114.

Cosic I. 1995. Virtual spectroscopy for fun and profit. Biotechnology 13:236-238.

Cosic I. 1997. The Resonant Recognition Model of Macromolecular Bioactivity: Theory and Applications. Basel: Birkhauser Verlag.

Cosic I, Cosic D, Lazar K. 2015. Is it possible to predict electromagnetic resonances in proteins, DNA and RNA?. Nonlinear Biomedical Physics 3.

Cosic I, Cosic D, Lazar K. 2016. Environmental Light and Its Relationship with Electromagnetic Resonances of Biomolecular Interactions, as Predicted by the Resonant Recognition Model. International Journal of Environmental Research and Public Health 13(7):647.

Cosic I, Cosic D. 2016. The Treatment of CriglerNajjar Syndrome by Blue Light as

Explained by Resonant Recognition Model. EPJ Nonlinear Biomedical Physics 4(9).

Dotta BT, Murugan NJ, Karbowski LM, Lafrenie RM, Persinger MA. 2014. Shifting wavelength of ultraweak photon emissions from dying melanoma cells: their chemical enhancement and blocking are predicted by Cosic's theory of resonant recognition model for macromolecules. Naturwissenschaften 101(2).

Gomes-Alves P, Neves S, Coelho AV, Penque D. 2009. Low Temperature Restoring Effect on F508del-CFTR Misprocessing: A Proteomic Approach. J Proteomics 73(2):218-230.

Karbowski LM, Murugan NJ, Persinger MA. 2015. Novel Cosic resonance (standing wave) solutions for components of the JAK-STAT cellular signalling pathway: A convergence of spectral density profiles. FEBS Open Bio 5:245-250.

Murugan NJ, Karbowski LM, Persinger MA. 2014. Cosic's Resonance Recognition Model for Protein Sequences and Photon Emission Differentiates Lethal and Non-Lethal Ebola Strains: Implications for Treatment. Open Journal of Biophysics 5:35.

O'Sullivan BP, Freedman SD. 2009. Cystic Fibrosis. Lancet 373(9678):1891-1904.

Pirogova E, Cosic I. 2002. The Use of Ionisation Constants of Amino Acids for Protein Signal Analysis within the Resonant Recognition Model Application to Proteases. Molecular Simulation 28(8):853-863.

Pranke I, Golec A, Hinzpeter A, Edelman A, SermetGaudelus I. 2019. Emerging Therapeutic Approaches for Cystic Fibrosis from Gene Editing to Personalized Medicine. Frontiers in Pharmacology 10(121).

Rennolds J, Boyaka PN, Bellis SL, Cormet-Boyaka E. 2008. Low Temperature Induces the Delivery of Mature and Immature CFTR to the Plasma Membrane. Biochemical and Biophysical Research Communications 366(4):1025-1029.

Schultz A, Marsh JA, Saville BR, Norman R, Middleton PG, Greville HW, Bellgard MI, Berry SM, Snelling T. 2019 Trial Refresh: A Case for an Adaptive Platform Trial for Pulmonary Exacerbations of Cystic Fibrosis. Frontiers in Pharmacology 10(301).

Vojisavljevic V, Pirogova E, Cosic I. 2007. The Effect of Electromagnetic Radiation (550nm-850nm) on I-Lactate Dehydrogenase Kinetics. Internat J Radiat Biol 83:221-230.

Wilke M, Bot A, Jorna H, Scholte BJ, de Jonge HR. 2012. Rescue of Murine F508del CFTR Activity in Native Intestine by Low Temperature and Proteasome Inhibitors. PLOSOne.

\section{Appendix \\ Resonant Recognition Model}

Here, we present our own nonconventional, biophysical, theoretical Resonant Recognition Model (RRM), which is based on the findings that certain periodicities within the distribution of energy of delocalized electrons along protein molecules are critical for protein biological functions and/or interactions with their targets ${ }^{(1-12)}$. The RRM model has been extensively published and experimentally successfully tested ${ }^{(1-28)}$. The RRM model has been presented and well explained in our previous publications as follows:

All proteins can be considered as a linear sequence of their constitutive elements, i.e. amino acids and 
biological function of proteins is determined primarily by this linear sequence. The RRM ${ }^{(1-3)}$ interprets this linear information by transforming protein sequence into a numerical series and then into the frequency domain using digital signal processing method: Fast Fourier Transform (FFT).

Protein primary structure can be presented as a numerical series by assigning the relevant physical parameter value to each amino acid. Our investigations have shown that the best correlation can be achieved with parameters which are related to the energy of delocalised electrons of each amino acid (calculated as Electron Ion Interaction Potential (EIIP)), as electrons delocalised from the particular amino acid, have the strongest impact on the electronic distribution of the whole protein ${ }^{(1-4)}$. The resulting numerical series represents the distribution of the free electron's energies along the protein molecule.

Such numerical series are then analysed by digital signal analysis methods, using FFT, to extract information pertinent to the biological function. As the distance between amino acid residues in a polypeptide chain (protein backbone) is $3.8 \AA$, it can be assumed that the points in the numerical sequence are equidistant. For further numerical analysis, the distance between points in these numerical sequences is set at an arbitrary value of $d=1$. Therefore, the maximum frequency in the spectrum is $\mathrm{F}=1 / 2 \mathrm{~d}=0.5$. The total number of points in the sequence influences the resolution of the spectrum only. Thus, for $\mathrm{N}$-point sequence the resolution in the spectrum is equal to $1 / \mathrm{N}$. The $n$-th point in the spectral function corresponds to the frequency $\mathrm{f}=\mathrm{n} / \mathrm{N}$.

To extract common spectral characteristics of sequences having the same or similar biological function, the multiple cross-spectral function is used. Peak frequencies in such a multiple cross-spectral function present common frequency component for all sequences analysed. Such common frequency components are found to be related to the common biological function of the analysed proteins leading to the conclusion that each specific biological function within the protein is characterised by one frequency $(1-3,5-6)$

Each biological function and/or process is driven by proteins that selectively interact with other proteins, DNA regulatory segments or small molecules. Using the RRM, it has been shown that proteins and their targets share the same matching characteristic frequency (periodicity) ${ }^{(1-3,5-6)}$. The matching of periodicities within the distribution of energies of free electrons along the interacting proteins can be regarded as the resonant recognition and is highly selective. Thus, the RRM frequencies characterise not only protein function, but also recognition and interaction between a protein and its targets: receptors, binding proteins and inhibitors. In addition, it has been also shown that interacting proteins have opposite phases at their characteristic recognition frequency ${ }^{(1-3,5,7)}$. Every frequency can be presented by one sinusoid characterised with its frequency, amplitude and phase. The phase is presented in radians and can be between $-\pi$ and $+\pi(-3.14$ and $+3.14)$. The phase difference of or about 3.14 is considered opposite phase. The phase value can be presented in the phase circle where it is easier to observe graphically opposite phases.

It has been proposed that the RRM frequencies characterize, not only a general function, but also a recognition and interaction between the macromolecule and its target, which then can be considered as resonant recognition. This could be achieved with resonant energy transfer between the interacting macromolecules through oscillations of a physical field, which is electromagnetic in nature. Since there is evidence that proteins have certain conducting or semi-conducting properties, a charge moving through the macromolecular backbone and passing different energy stages, caused by different amino acid or nucleotide side groups, can produce sufficient conditions for a specific electromagnetic radiation or absorption. The frequency ranges of this field depend on the charge velocity. The RRM proposes, based on solid state physics principles, that the charge is travelling through the macromolecular backbone at the estimated velocity of $7.87 \times 10^{5} \mathrm{~m} / \mathrm{s}$ ${ }^{(8,9)}$. For this velocity and with the distance between amino acids in a protein backbone of $3.8 \AA$, the frequency of protein interactions was estimated to be in the range between $10^{13} \mathrm{~Hz}$ and $10^{15} \mathrm{~Hz}$. Therefore, the estimated frequency range for both amino acid and nucleotide macromolecules includes far infra-red, infra-red, visible and ultra-violet light. To support this idea, we compared our RRM computational predictions for variety of biological functions with number of published experimental results ${ }^{(1-3)}$ :

- Laser light growth promotion of cells, by using the frequencies of light to produce the similar effect to that of growth factor proteins;

- Chymotrypsin activation (increase of enzyme activity) achieved by laser light radiation in a range of $850-860 \mathrm{~nm}$;

- Activation of highly homologous plant photoreceptors which, although being very homologous, absorb different wavelengths of light;

- Photo activated proteins, e.g. rhodopsin, flavodoxin, etc.

These comparisons have empirically shown a strong linear correlation between frequencies, as calculated using the RRM method and experimentally measured characteristic frequencies, with the slope factor of $\mathrm{K}=201^{(1-3,10)}$. This finding parallel with the frequency range previously associated with the RRM numerical frequency spectrum that has been calculated from the charge velocities through the protein backbone. This 
correlation can be represented as following:

$$
\lambda=\mathrm{K} / \mathrm{f}_{\text {rrm }}
$$

where $\lambda$ is the wavelength of light irradiation in $\mathrm{nm}$, which can influence particular biological process, $\mathrm{f}_{\text {rrm }}$ is RRM numerical frequency and $K$ is coefficient of this linear correlation.

We applied this concept on number of proteins and DNA examples ${ }^{(4-15)}$. The concept has been also experimentally tested by predicting the electromagnetic frequencies for activation of $\mathrm{L}$ Lactate Dehydrogenase ${ }^{(12)}$. In this experiment, by radiating L-Lactate Dehydrogenase with RRM predicted calculated electromagnetic frequencies the significant change in enzyme activity was achieved. The concept has also been tested independently on experimental measurements of photon emission from dying melanoma cells ${ }^{(13)}$, on photon emission from lethal and non-lethal Ebola strains ${ }^{(14)}$, as well as on classic signalling pathway, JAK-STAT, traditionally composed of nine sequential protein interactions ${ }^{(15)}$. Even more, the RRM model, for the first time, explains how and why external blue light can be used in the treatment of Crigler-Najjar syndrome ${ }^{(11)}$.

Keeping all this in mind, we propose that the RRM concept is excellent predictor for proteins selective interactions, biological processes and pathways in living cells. In our previous work, we have calculated large number of specific frequencies for different protein and DNA biological functions and interactions ${ }^{(1-12,16-23)}$.

Bioactive peptide design

Once the characteristic biological function of the protein is identified, it is possible to design new proteins with desired frequency components and consequently with desired biological functions ${ }^{(1-3,7}$, 17-23). The process of bioactive peptides design is as follows:

- Determination of RRM characteristic frequency using multiple cross-spectral function for a group of protein sequences that share common biological function (interaction);

- Determination of phases for the characteristic frequencies of a particular protein which is selected as the parent for agonist/antagonist peptide;

- Calculation using Inverse Fourier Transform of the signal with characteristic frequency and phase. The minimal length of the designed peptide is defined by the characteristic frequency $\mathrm{f}$ as $1 / \mathrm{f}$;

- Determination of resulting amino acid sequence using tabulated EIIP parameter values.

This approach has been already successfully applied and experimentally tested in design of FGF $(1-3,18)$, HIV envelope protein analogue ${ }^{(1-3,7,19-20)}$, mice IL-12 analogue ${ }^{(21)}$ and peptide to mimic myxoma virus oncolytic function ${ }^{(22-23)}$.
It is interesting to note that de novo designed peptides do not have any significant homology with the original protein, but when their 3D structure is predicted it has been shown that this 3D structure is very similar to $3 \mathrm{D}$ structure of original protein active site ${ }^{(18)}$. As 3D structure is defined by protein primary structure, it seems that RRM by analysing frequencies within primary structure can decipher rules how 3D structure is formed.

Prediction of the key amino acids - "hot spots"

Knowing the characteristic frequency of particular protein function creates the possibility to predict which amino acids prevail in the sequence and predominantly contribute to this frequency and consequently to the observed function. This could be achieved by small alternations of amplitude in single protein spectrum at characteristic frequency and then observing which amino acids are mostly sensitive to this alternation ${ }^{(1-3,24-28)}$. These sensitive amino acids ("hot spots") are related to characteristic frequency and consequently to the corresponding biological function. The "hot spots" predictions, using the RRM, have been applied already to number of protein and DNA examples including: interleukin-2, SV40 enhancer, epidermal growth factor EGF, Ha-ras p21 oncogene product, glucagons, haemoglobins, myoglobins and lysozymes $(1-3,24-28)$. It has been experimentally documented at the example of influenza virus that such predicted amino acids denote residues crucial for protein function ${ }^{(28)}$.

In addition, these "hot spots" amino acids, although not sequentially linked, are found to be spatially clustered in the protein tertiary structure and to be positioned in and around the protein active site ${ }^{(24-28)}$. This could mean that protein 3D structure is formed as resonant box for the characteristic frequency.

Running Title: Electromagnetic Resonances in CFTR Proteins

Conflict of Interest: none

This research did not receive any specific grant from funding agencies in the public, commercial, or notfor-profit sector

\section{Reference}

1. Cosic I: Macromolecular Bioactivity: Is it Resonant Interaction between Macromolecules? -Theory and Applications. IEEE Trans on Biomedical Engineering, 1994, 41, pp. 1101-1114.

2. Cosic I: Virtual spectroscopy for fun and profit. Biotechnology, 1995, 13, pp. 236-238.

3. Cosic I: The Resonant Recognition Model of Macromolecular Bioactivity: Theory and Applications. Basel: Birkhauser Verlag, 1997.

4. Pirogova E, Cosic I: Examination of amino acid indexes within the Resonant Recognition Model. Proc. of the 2nd Conference of the Victorian Chapter of the IEEE EMBS, 2001, pp. 124-127.

5. Cosic I, Cosic D, Lazar K: Analysis of Tumor Necrosis Factor Function Using the Resonant Recognition Model. Cell Biochemistry and Biophysics, 2015, 11, doi: 10.1007/s12013-015-0716-3.

6. Cosic I, Lazar K, Cosic D: Cellular Ageing - Telomere, 
Telomerase and Progerin analysed using Resonant Recognition Model. MD-Medical Data, 2014, 6(3), pp. 205209.

7. Krsmanovic V, Biquard JM, Sikorska-Walker M, Cosic I, Desgranges C, Trabaud MA, Whitfield JF, Durkin JP, Achour A, Hearn MT: Investigation into the Cross-reactivity of Rabbit Antibodies Raised against Nonhomologous Pairs of Synthetic Peptides Derived from HIV-1 gp120 proteins. J. Peptide Res, 1998, 52(5), pp. 410-412.

8. Cosic I, Lazar K, Cosic D: Prediction of Tubulin resonant frequencies using the Resonant Recognition Model (RRM). IEEE Trans. on NanoBioscience, 2015, 12, pp. 491-496, doi: 10.1109/TNB.2014.2365851.

9. Cosic I, Cosic D, Lazar K: Is it possible to predict electromagnetic resonances in proteins, DNA and RNA? Nonlinear Biomedical Physics, 2015, 3, doi: 10.1140/s40366015-0020-6.

10. Cosic I, Cosic D, Lazar K: Environmental Light and Its Relationship with Electromagnetic Resonances of Biomolecular Interactions, as Predicted by the Resonant Recognition Model. International Journal of Environmental Research and Public Health, 2016, 13(7), pp. 647, doi: 10.3390/ijeprh13070647.

11. Cosic I, Cosic D: The Treatment of Crigler-Najjar Syndrome by Blue Light as Explained by Resonant Recognition Model. EPJ Nonlinear Biomedical Physics, 2016, 4(9), doi: 10.1140/epjnbp/s40366-016-0036-6.

12. Vojisavljevic V, Pirogova E, Cosic I: The Effect of Electromagnetic Radiation (550nm-850nm) on I-Lactate Dehydrogenase Kinetics. Internat J Radiat Biol, 2007, 83, pp. 221-230.

13. Dotta BT, Murugan NJ, Karbowski LM, Lafrenie RM, Persinger MA: Shifting wavelength of ultraweak photon emissions from dying melanoma cells: their chemical enhancement and blocking are predicted by Cosic's theory of resonant recognition model for macromolecules. Naturwissenschaften, 2014, 101(2), doi: 10.1007/s00114013-1133-3.

14. Murugan NJ, Karbowski LM, Persinger MA: Cosic's Resonance Recognition Model for Protein Sequences and Photon Emission Differentiates Lethal and Non-Lethal Ebola Strains: Implications for Treatment. Open Journal of Biophysics, 2014, 5, pp. 35.

15. Karbowski LM, Murugan NJ, Persinger MA: Novel Cosic resonance (standing wave) solutions for components of the JAK-STAT cellular signalling pathway: A convergence of spectral density profiles. FEBS Open Bio, 2015, 5, pp. 245250.

16. Pirogova E, Istivan T, Gan E, Cosic I: Advances in methods for therapeutic peptide discovery, design and development, Current Pharmaceutical Biotechnology. Bentham Science
Publishers Ltd, Netherlands ISSN: 1389-2010, 2011, 12(8), pp. 1117-1127.

17. Cosic I, Pirogova E: Bioactive Peptide Design using the Resonant Recognition Model. Nonlinear Biomedical Physics, 2007, 1(7), doi: 10.1186/1753-4631-1-7.

18. Cosic I, Drummond AE, Underwood JR, Hearn MTW: In vitro inhibition of the actions of basic FGF by novel 16 amino acid peptides. Molecular and Cellular Biochemistry, 1994, 130, pp. 1-9.

19. Hearn MTW, Biquard JM, Cosic I, Krsmanovic V: Peptides Immunologically related to proteins expressed by a viral agent, having a sequence of amino acids ordered by means of protein informational method. US Patent 6, 294, 174, 2001.

20. Achour A, Biquard JM, Krsmanovic V, M'Bika JP, Ficheux D, Sikorska M, Cozzone AJ: Induction of Human Immunodeficiency Virus (HIV-1) Envelope Specific CellMediated Immunity by a Non-Homologus Synthetic Peptide. PLoS ONE, 2007, 11, pp. 1-12, doi: 10.1371/journal.pone.0001214.

21. Pirogova E, Istivan T, Gan E, Cosic I: Advances in Methods for Therapeutic Peptide Discovery, Design and Development. Current Pharmaceutical Biotechnology, 2011, 12, pp. 11171127.

22. Almansour N, Pirogova E, Coloe P, Cosic I, Istivan T: Investigation of cytotoxicity of negative control peptides versus bioactive peptides on skin cancer and normal cells: a comparative study. Future Medicinal Chemistry, 2012, 4(12), pp. 1553-1565.

23. Istivan T, Pirogova E, Gan E, Almansour N, Coloe P, Cosic I: Biological effects of a De Novo designed myxoma virus peptide analogue: Evaluation of cytotoxicity on tumor cells. Public Library of Science (PLoS) ONE, 2011, 6(9), pp. 1-10.

24. Cosic I, Hearn MTW: "Hot Spot" Amino Acid Distribution in Ha-ras Oncogene Product p21: Relationship to Guanine Binding Site. J. Molecular Recognition, 1991, 4, pp. 57-62.

25. Cosic I, Hearn MTW: Studies on Protein-DNA Interactions Using the Resonant Recognition Model: Application to Repressors and Transforming Proteins. Eur. J. Biochem, 1992, 205, pp. 613-619.

26. Cosic I, Hodder AN, Aguilar MI, Hearn MTW: Resonant Recognition Model and protein topography: model studies with myoglobin, haemoglobin and lysozyme. Eur. J. Biochem, 1991, 198, pp. 113-119.

27. Caceres JLH, Cosic I, Cosic D: Application of The Resonant Recognition Model to The Study of Plasmodium Proteins Involved in Malaria Infection. MD-Medical Data, 2015, 7(1), pp. 007-014.

28. Schmier S, Mostafa A, Haarmann T, Bannert N, Ziebuhr J, Veljkovic V, Dietrich U, Pleschka S: In silico prediction and experimental confirmation of $\mathrm{HA}$ residues conferring enhanced human receptor specificity of $\mathrm{H} 5 \mathrm{~N} 1$ influenza A viruses. Scientific Reports, 2015, 5, doi: 10.1038/srep11434. 